\title{
Overexpression of miRNA-22-3p attenuates osteoporosis by targeting MAPK14
}

\author{
XIAOLIN JIA ${ }^{1}$, MING YANG $^{1}$, WEI HU $^{1}$ and SAN CAI $^{2}$ \\ ${ }^{1}$ Department of Orthopaedics, Chongqing General Hospital, Chongqing 401147; \\ ${ }^{2}$ Department of Orthopaedics, Chongqing Public Health Medical Center, Chongqing 400036, P.R. China
}

Received October 21, 2020; Accepted March 29, 2021

DOI: $10.3892 /$ etm.2021.10124

\begin{abstract}
Osteoporosis (OP) results from an imbalance between bone formation, which is regulated by osteoblasts, and bone resorption, which is mediated by osteoclasts. MicroRNA-22-3p (miR-22-3p) expression is decreased during the process of osteoclast differentiation and $\mathrm{p} 38 \alpha$ mitogen-activated protein kinase (MAPK)14 promotes the proliferation and differentiation of osteoclast progenitors. However, whether miR-22-3p could target MAPK14 to regulate the progression of OP remains unknown, which was the aim of the present study. CD14+ PBMCs were used for the establishment of osteoclastic differentiation in vitro. In the present study, reverse transcription quantitative PCR was used to determine the mRNA expression of MAPK14, tartrate resistant acid phosphatase (TRAP), nuclear factor of activated T-cells (NFATC1) and cathepsin K (CTSK). Western blotting was applied to determine the protein expression of MAPK14, TRAP, NFATC1, CTSK, p-p65 and p65. Dual luciferase reporter assay was applied to confirm the relation between miR-22-3p and MAPK14. Cell Counting Kit-8 assay and flow cytometry assays were used to determine the cell proliferation and cell apoptosis, respectively. The results demonstrated that miR-22-3p expression was lower while MAPK14 expression was higher in the serum from patients with OP compared with healthy volunteers. Furthermore, miR-22-3p expression was negatively correlated with MAPK14 expression in patients with OP. In addition, miR-22-3p expression was decreased and MAPK14 expression was increased during the progression of $\mathrm{CD} 14^{+}$peripheral blood mononuclear cells (PBMCs) osteoclastic differentiation in a time-dependent manner. Furthermore, miR-22-3p inhibited the proliferation and differentiation and promoted the apoptosis of CD14+PBMCs by targeting MAPK14. In summary, the findings from the
\end{abstract}

Correspondence to: Dr San Cai, Department of Orthopaedics, Chongqing Public Health Medical Center, 109 Baoyu Road, Chongqing 400036, P.R. China

E-mail: caisancqphmc@outlook.com

Key words: osteoporosis, microRNA-22-3p, mitogen-activated protein kinase 14 present study suggested that miR-22-3p may serve a potential therapeutic role in patients with OP.

\section{Introduction}

Osteoporosis (OP) remains the most common progressive skeletal disease. This disease limits the activity of patients (1) and decreases the quality of life in elders and postmenopausal women (2). The modeling and remodeling of bone is a dynamic metabolic process primarily mediated by the osteoblasts, which form new bone by secretion of bone matrix and acceleration of calcium $\left(\mathrm{Ca}^{2+}\right)$ deposition, and the osteoclasts, which resorb old bone by resolving mineralized bone matrix $(3,4)$. In case of imbalance between osteoblastic bone formation and osteoclastic bone resorption (5), which can lead to upregulated bone resorption, downregulated bone formation or both $(6,7)$, OP can occur.

MicroRNAs (miRNAs) are a subclass of non-coding RNAs of 19-25 nucleotides in length, which regulate the expression of target genes at post-transcriptional level (8). Previous studies have reported the importance of miRNAs in the regulation of skeletal development, bone formation and homeostasis $(9,10)$. For example, miR-125b, miR-29a and miR-378 have been demonstrated to be involved in osteoblastic differentiation (11-13). Furthermore, miR-21, miR-155 and miR-223 have been found to be implicated in osteoclastic differentiation $(14,15)$. In 2015, miR-22-3p expression was found to be decreased in the bone of patients who suffered a bone fracture (16). In 2016, miR-22-3p was discovered to be downregulated during the progression of osteoclastic differentiation (17). In 2018, miR-22-3p expression was reported to be decreased in the bone of patients with OP (18). In 2020, extracellular vesicle-encapsulated miR-22-3p from bone marrow mesenchymal stem cells were found to induce osteogenic differentiation via Fat mass- and obesity-associated gene inhibition (19). However, the underlying mechanisms of miR22-3p during the process of osteoclast differentiation have not yet been reported, which was explored in the present study.

\section{Materials and methods}

Clinical samples. A total of 30 healthy volunteers (mean age, $59.79 \pm 6.53$ years; age range, $47-68$ years) and 30 postmenopausal women with OP (mean age, $60.23 \pm 7.15$ years; age range, 
49-69 years) were included in the present study. Blood samples $(5 \mathrm{ml})$ were collected from each participant for the determination of miR-22-3p and p38 $\alpha$ mitogen-activated protein kinase (MAPK)14 expression. This study was approved by the Ethics Committee of Chongqing Public Health Medical Center (approval no. CMC20180106). Each participant provided a signed informed consent prior to the beginning of the study.

Bone mineral density (BMD) measurement. The BMD of each participant was determined at the lumbar spine (L1-L4) and the left femoral neck through dual-energy X-ray absorptiometry (DXA) using a Hologic 4500 bone densitometer (Hologic), according to the World Health Organization criteria (20). Each scan was handled by a single technician and repeated thrice with repositioning between each scan. The precision of the machine, which is presented as percentage coefficient of variation (CV\%), varied between subregions. CV\% for BMD of the lumbar spine (L1-L4) was $<1 \%$, whereas $\mathrm{CV} \%$ for BMD of the left femoral was $<2 \%$.

Isolation and incubation of $\mathrm{CD} 4^{+}$peripheral blood mononuclear cells (PBMCs). Osteoclasts are primary bone-resorbing cells which can form from precursor fusion, and CD14 ${ }^{+} \mathrm{PBMCs}$ are early progenitors for osteoclasts (21). Furthermore, osteoclast formation can occur in $\mathrm{CD} 14^{+} \mathrm{PBMCs}$ with the stimulation of macrophage colony stimulating factor (M-CSF) and receptor activator of nuclear factor- $\kappa \mathrm{B}$ ligand (RANKL) (22). Subsequently, the present study used CD14+PBMCs for the study of OP in vitro. PBMCs were extracted as previously described (22). CD14+PBMCs were purified using CD14 antibody-coated magnetic cell sorting MicroBeads (Miltenyi Biotec $\mathrm{GmbH}$ ). When CD14+PBMCs reached $90 \%$ purity by flow cytometry, they were seeded into 48 -well plates at the density of $2.5 \times 10^{5}$ cells/well. CD14 ${ }^{+}$PBMCs were incubated in $\alpha$-MEM (Invitrogen; Thermo Fisher Scientific, Inc.) containing 10\% FBS (Gibco; Thermo Fisher Scientific, Inc.), penicillin $(50 \mathrm{IU} / \mathrm{ml})$ and streptomycin $(50 \mathrm{mg} / \mathrm{ml})(\mathrm{Gibco}$; Thermo Fisher Scientific, Inc.) and placed in an incubator with $5 \% \mathrm{CO} 2$ at $37^{\circ} \mathrm{C}$ for $2 \mathrm{~h}$.

Osteoclastic differentiation. For the establishment of osteoclastic differentiation in vitro, $\mathrm{CD} 14^{+} \mathrm{PBMC}$ initial complete culture medium was changed for $\alpha$-MEM containing M-CSF (25 ng/ml; R\&D Systems, Inc.) and RANKL (25 ng/ml; R\&D Systems, Inc.). The complete culture medium containing differentiation factors, $10 \% \mathrm{FBS}$, penicillin $(50 \mathrm{IU} / \mathrm{ml})$ and streptomycin $(50 \mathrm{mg} / \mathrm{ml})$ was refreshed every three days and floating cells were removed. After incubation for six days, CD14 ${ }^{+}$PBMCs were collected for subsequent experiments.

Cell transfection. For the overexpression of miR-22-3p, CD14 ${ }^{+}$PBMCs $\left(2 \times 10^{4}\right.$ cells $\left./ \mathrm{ml} ; 200 \mu \mathrm{l}\right)$ were seeded into 48-well plates and transfected with miR-NC mimic (sense, 5'-UUCUCCGAACGUGUCACGU-3' and antisense, 5'-ACGUGACACGUUCGGAGAA-3') or miR-22-3p mimic (sense, 5'-AAAAGCUGCCAGUUGAAGAACUGU-3' and antisense, 5'-ACAGUUCUUCAACUGGCAGCUUUU-3') (50 nmol/1; Guangzhou Ribobio Co., Ltd.) using Lipofectamine ${ }^{\circledR} 2000$ (Invitrogen; Thermo Fisher Scientific, Inc.). After transfection for $48 \mathrm{~h}, \mathrm{CD} 14^{+} \mathrm{PBMCs}$ were subsequently
Table I. Clinicopathological characteristics of participants.

\begin{tabular}{lccc}
\hline Variable & $\begin{array}{c}\text { Healthy } \\
\text { volunteers }\end{array}$ & $\begin{array}{l}\text { Patients } \\
\text { with OP }\end{array}$ & P-value \\
\hline Age, years & $59.79 \pm 6.53$ & $60.23 \pm 7.15$ & $>0.05$ \\
BMI, kg/m² & $23.59 \pm 4.14$ & $24.23 \pm 3.09$ & $>0.05$ \\
Femoral neck & $0.89 \pm 0.12$ & $0.62 \pm 0.13$ & $<0.001$ \\
BMD, g/cm & & & \\
Lumbar spine & $0.85 \pm 0.16$ & $0.61 \pm 0.09$ & $<0.001$ \\
BMD, g/cm & & & \\
\hline
\end{tabular}

treated with M-CSF and RANKL as aforementioned and collected for subsequent experiments.

To overexpress MAPK14, CD14 PBMCs $\left(2 \times 10^{4}\right.$ cells $/ \mathrm{ml}$; $200 \mu \mathrm{l}$ ) were seeded into 48-well plates and transfected with pcDNA3.1 or pcDNA3.1-MAPK14 (2 $\mu \mathrm{g}$; Sangon Biotech Co., Ltd.) using Lipofectamine 2000. After transfection for 48 h, CD14+PBMCs were subsequently treated with M-CSF and RANKL as aforementioned and collected for subsequent experiments. At $48 \mathrm{~h}$ after the transfection, cells were used for subsequent experiments.

Dual luciferase reporter assay. The binding site between miR-22-3p and MAPK14 was predicted by TargetScan 7.1 (http://www.targetscan.org/vert_71/). To verify the interaction between miR-22-3p and MAPK14, CD14+PBMCs $\left(1 \times 10^{5}\right.$ cells $\left./ \mathrm{ml}\right)$ were seeded into $24-w e l l$ plates and co-transfected with wild type (WT) or mutant (MUT) MAPK14 3'-UTR (400 ng) which was cloned into psiCHECK2 (Promega Corporation) and miR-NC mimic or miR-22-3p mimic (20 nmol/l) using Lipofectamine 2000. After incubation for $48 \mathrm{~h}, \mathrm{CD} 14^{+} \mathrm{PBMCs}$ were collected for the determination of luciferase activity with the dual-luciferase reporter assay (Promega Corporation). The relative luciferase activity was normalized to Renilla luciferase activity (Promega corporation).

Cell proliferation assay. CD14 ${ }^{+} \mathrm{PBMC}$ proliferation was determined using Cell Counting Kit-8 (CCK-8; Dojindo Molecular Technologies, Inc.). CD14+PBMCs were seeded into 96-well (1x10 $3 /$ well) and incubated for $0,24,48$ or 72 h. CCK-8 solution $(10 \mu \mathrm{l})$ was added into each well and incubated for $1 \mathrm{~h}$ at $37^{\circ} \mathrm{C}$. Absorbance at $450 \mathrm{~nm}$ was determined on a microplate reader.

Cell apoptosis assay. CD14+PBMC cell apoptosis was determined using the Annexin V-FITC/propidium iodide (PI) Apoptosis Detection kit (Sigma-Aldrich; Merck KGaA) on a flow cytometer (BD Biosciences). Briefly, CD14+PBMCs were collected by after centrifugation at $1,000 \mathrm{x}$ g for $5 \mathrm{~min}$ at $4^{\circ} \mathrm{C}$ and resuspended in $500 \mu \mathrm{l}$ binding buffer. Subsequently, CD14 ${ }^{+}$PBMCs were stained with Annexin V-FITC (100 $\left.\mu \mathrm{l}\right)$ for $15 \mathrm{~min}$ and PI $(10 \mu \mathrm{l})$ for $5 \mathrm{~min}$ in the dark. The cell apoptotic rate was quantified on a flow cytometry and analyzed by CellQuest version 3.3 (BD Biosciences).

RNA isolation from serum and cells. Serum samples were obtained from whole blood by centrifugation at $2,000 \mathrm{x} \mathrm{g}$ 

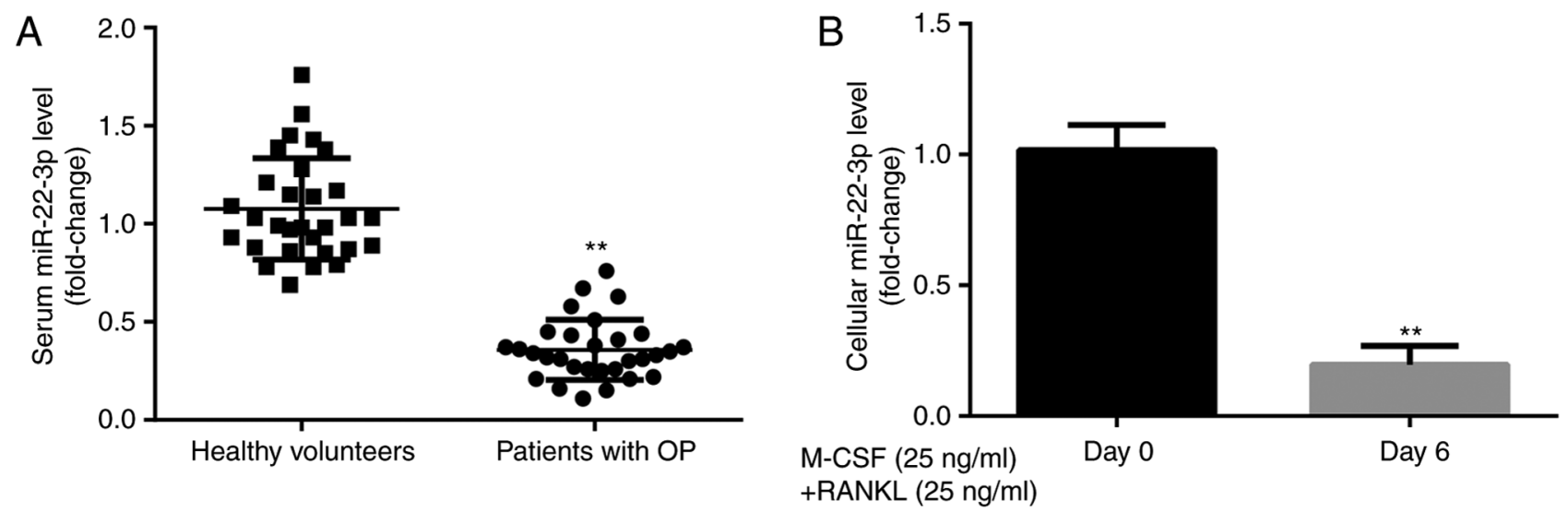

Figure 1. miR-22-3p expression level is decreased in serum and CD14+PBMCs from patients with OP. miR-22-3p expression was lower in (A) patients with postmenopausal OP compared with healthy volunteers and (B) CD14+PBMCs treated with M-CSF and RANKL for 6 days compared with untreated cells. ${ }^{* *} \mathrm{P}<0.01$. OP, osteoporosis; miR, microRNA; PBMCs, peripheral blood mononuclear cells; M-CSF, macrophage colony stimulating factor; RANKL, receptor activator of nuclear factor- $\mathrm{\kappa} B$ ligand.

for $15 \mathrm{~min}$ at room temperature after being incubated at room temperature for $30 \mathrm{~min}$. For detection of miRNA, total RNA was isolated from serum $(200 \mu \mathrm{l})$ of participants or CD14+PBMCs using miRNeasy Serum/Plasma kit (Qiagen $\mathrm{GmbH}$ ). For the detection of mRNA, total RNA was isolated from serum of participants or CD14 ${ }^{+}$PBCs using TRIzol ${ }^{\circledR}$ (Invitrogen; Thermo Fisher Scientific, Inc.).

Reversetranscription quantitative (RT-q)PCR.Complementary DNA (cDNA) was obtained from miRNA and mRNA using TaqMan MicroRNA Reverse Transcription kit (Thermo Fisher Scientific, Inc.) and First Strand cDNA Synthesis Kit (Takara Bio, Inc.), respectively. RT-qPCR reactions were performed as follows: $95^{\circ} \mathrm{C}$ for $30 \mathrm{sec}$, followed by 40 cycles at $95^{\circ} \mathrm{C}$ for $5 \mathrm{sec}$ and at $60^{\circ} \mathrm{C}$ for $20 \mathrm{sec}$ using SYBR Green qPCR assay kit (Takara Bio, Inc.) on an ABI 7500 Real-Time PCR System (Applied Biosystems; Thermo Fisher Scientific, Inc.). The relative expression levels of miR-22-3p and of MAPK14, tartrate resistant acid phosphatase (TRAP), nuclear factor of activated T-cells (NFATC1) and cathepsin K (CTSK) were normalized to endogenous controls U6 or GAPDH, respectively, and were expressed as $2^{-\Delta \Delta \mathrm{Cq}}$ (23). The following primer sequences were used: GAPDH forward, 5'-GCACCGTCAAGGCTGAG AAC-3' and reverse, 5'-TGGTGAAGACGCCAGTGGA-3'; U6 forward, 5'-GTGCTCGCTTCGGCAGCACAT-3' and reverse, 5'-AATATGGAACGCTTCACGAAT-3'; CTSK forward, 5'-TCCGCAATCCTTACCGAATA-3' and reverse, 5'-AACTTGAACACCCACATCCTG-3'; NFATC1 forward, 5'-TACCAGCGTTTCACCTACCT-3' and reverse, 5'-СССТTTTССТTTCСТTТTCA-3'; TRAP forward, 5'-AGACATCAATGACAAGAGGT-3' and reverse, 5'-AAG TGCAGGCGGTAGAAAGG-3'; miR-22-3p forward, 5'-AAGCTGCCAGTTGAAGAACTGT-3' and reverse, 5'-ACAGTTCTTCAACTGGCAGCTT-3'; MAPK14 forward, 5'-GAAAAGGGTCTTCTTGGCAGCTT-3' and reverse, 5'-AAGCTGCCAAGAAGACCCTTTTC-3'.

Western blotting. CD14+PBMCs were lysed on ice using RIPA (Sigma-Aldrich; Merck KGaA) containing PMSF and protease inhibitors (Roche Diagnostics $\mathrm{GmbH}$ ). Protein concentration was determined by a BCA kit (Thermo Fisher Scientific, Inc.). Proteins (20 $\mu \mathrm{g}$ per lane) were separated by $10 \%$ SDS-PAGE and transferred onto PVDF membranes (EMD Millipore). Membranes were blocked with $5 \%$ bovine serum albumin (Beyotime Institute of Biotechnology) at room temperature for $1 \mathrm{~h}$ and were incubated with primary antibodies against GAPDH (1:1,000; cat. no. 5174; Cell Signaling Technology, Inc.), MAPK14 (1:1,000; cat. no. 8690; Cell Signaling Technology, Inc.), TRAP (1:1,000; cat. no. ab65854; Abcam), NFATC1 (1:1,000; cat. no. ab25916; Abcam), CTSK (1:1,000; cat. no. ab37259; Abcam), p-p65 (1:1,000; cat. no. 3033; Cell Signaling Technology, Inc.) and p65 (1:1,000; cat. no. 8242; Cell Signaling Technology, Inc.) at $4^{\circ} \mathrm{C}$ overnight. Membranes were then incubated with anti-rabbit $(1: 2,000$; cat. no. 7074$)$ or anti-mouse HRP-conjugated secondary antibody (1:2,000; cat. no. 7076; both from Cell Signaling Technology, Inc.) at room temperature for $1 \mathrm{~h}$. Enhanced chemiluminescence reagent (Bio-Rad Laboratories, Inc.) was used to detect the signal on the membrane. Densitometry analysis was performed using Image J software (v1.50; National Institutes of Health).

Statistical analyses. Statistical analyses were performed with the GraphPad Prism 6.0 (GraphPad Software, Inc.). Data were expressed as the means \pm standard deviation. Unpaired student's t-test was used to analyze differences between two groups from CD14+PBMCs and participants. One-way ANOVA followed by Tukey's post hoc test was used to analyze differences among multiple groups. Pearson correlation analysis was used to analyze the relationship between miR-22-3p and MAPK14 expression. $\mathrm{P}<0.05$ was considered to indicate a statistically significant difference.

\section{Results}

Clinicopathological characteristics of participants. The clinicopathological characteristics of participants are presented in Table I. There was no significant difference between the mean age of postmenopausal women with OP and healthy volunteers $(60.23 \pm 7.15$ years vs. $59.79 \pm 6.53$ years, respectively). The body mass indexes in the two groups were also not different 
A Position 1451-1457 of MAPK14 3'UTR 5'-GAAAAGGGUCUUCUU-GGCAGCUU Wild type hsa-miR-22-3p 3'------UGUCAAGAAGUUGACCCGUCGAA l| | |

Position 1451-1457 of MAPK14 3'UTR 5'-GAAAAGGGUCUUCUU--GGCCACUU Mutant
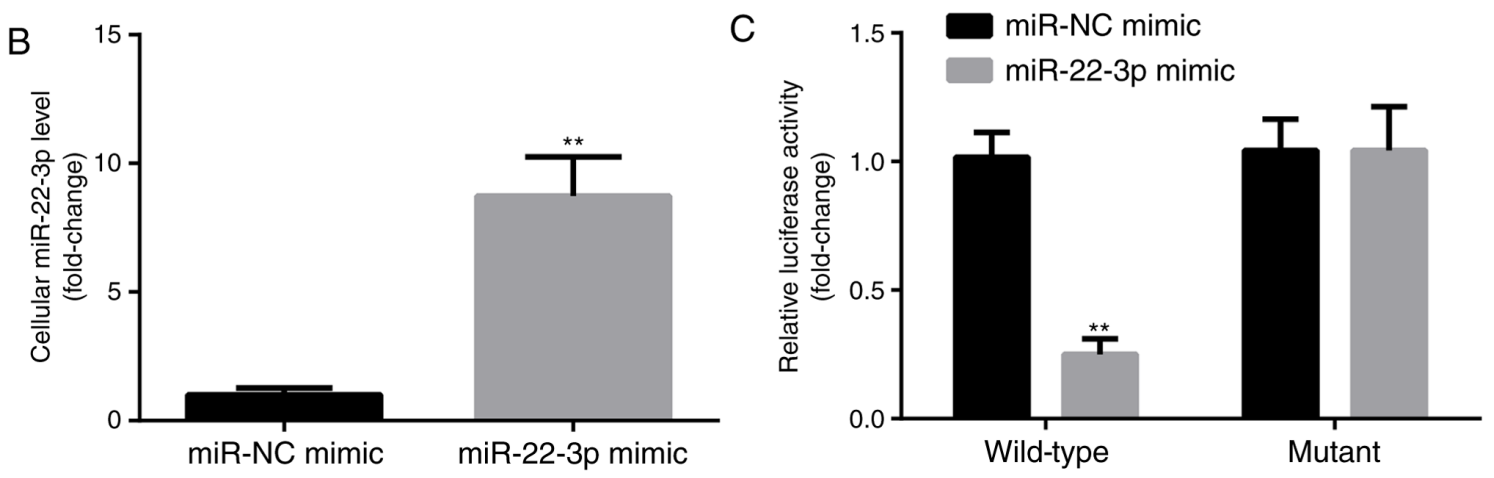

Figure 2. miR-22-3p targets the expression of MAPK14. (A) Binding sites between MAPK14 and miR-22-3p. (B) miR-22-3p mimic was successfully transfected into CD14+PBMCs. (C) miR-22-3p targeted WT-MAPK14. ${ }^{* *} \mathrm{P}<0.01$. miR, microRNA; PBMCs, peripheral blood mononuclear cells; NC, negative control.

A
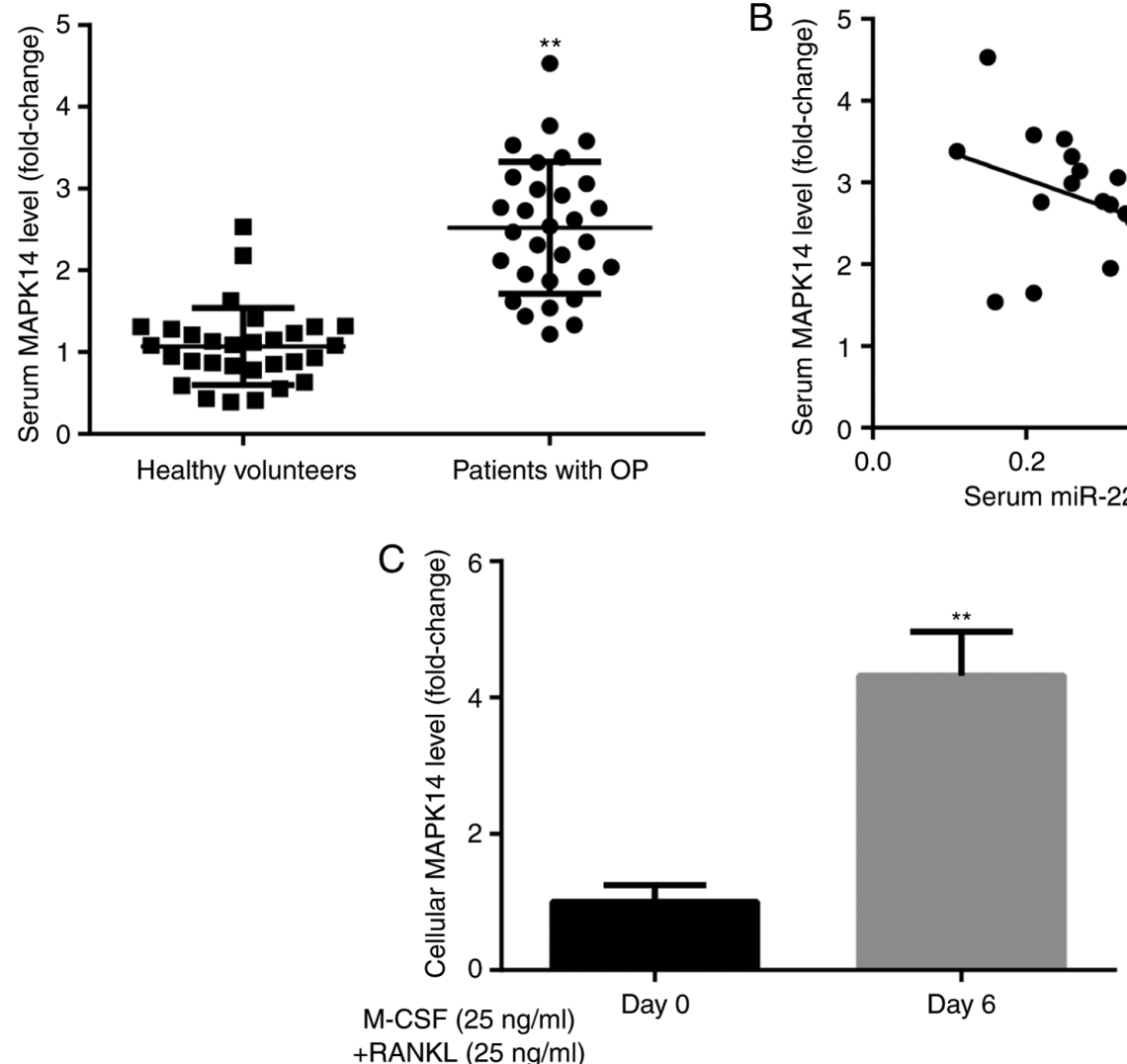

$\mathrm{B}$

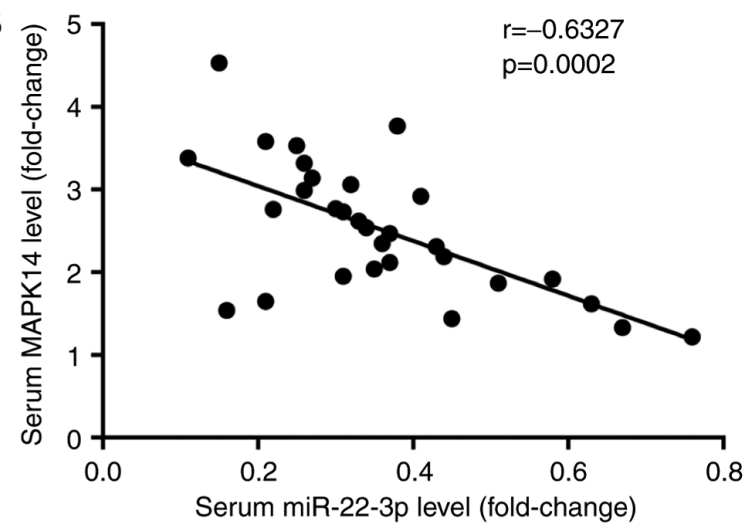

Figure 3. MAPK14 expression level is increased in serum and CD14 PBMCs from patients with OP.(A) MAPK14 expression was higher in patients with postmenopausal OP compared with healthy volunteers. (B) Negative correlation between miR-22-3p and MAPK14 expression. (C) MAPK14 expression was increased in CD14+PBMCs treated with M-CSF and RANKL for 6 days compared with untreated cells. "* $\mathrm{P}<0.01$. OP, osteoporosis; miR, microRNA; PBMCs, peripheral blood mononuclear cells; M-CSF, macrophage colony stimulating factor; RANKL, receptor activator of nuclear factor- $\mathrm{kB}$ ligand.

( $24.23 \pm 3.09$ vs. $23.59 \pm 4.14$ in postmenopausal women with $\mathrm{OP}$ and healthy volunteers, respectively). The mean femoral neck BMD and lumbar spine BMD $(0.62 \pm 0.13$ and $0.61 \pm 0.09$, respectively) of patients with postmenopausal OP were significantly lower compared with those of healthy volunteers $(0.89 \pm 0.12$ and $0.85 \pm 0.16$, respectively). The comparison of BMD was not adjusted for age and BMI according to a previous study (24).

miR-22-3p expression level in participants and $C D 14^{+} P B M C s$. To explore the effects of miR-22-3p during the development of OP, miR-22-3p expression level was evaluated in healthy 

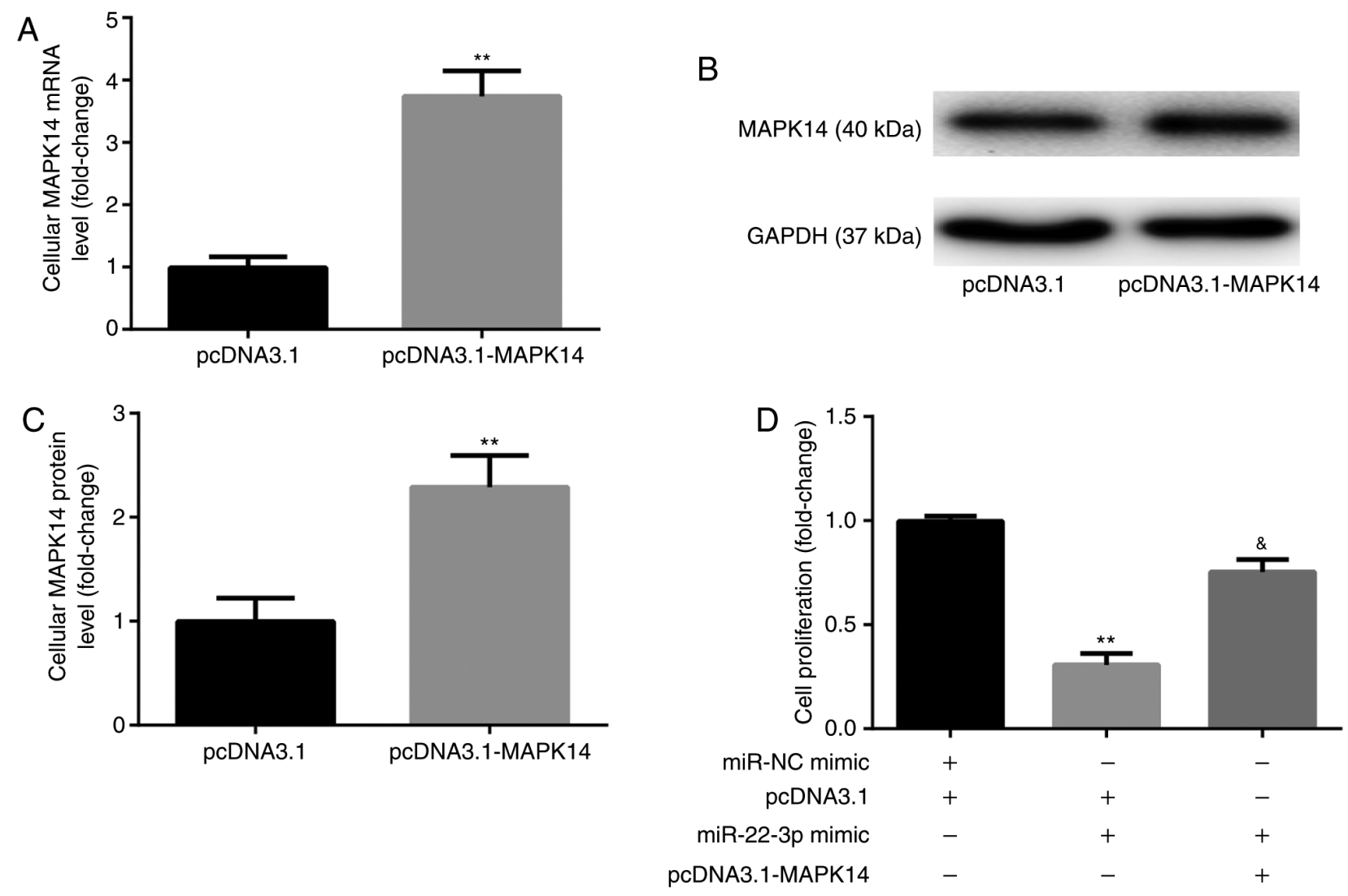

Figure 4. miR-22-3p mimic inhibits CD14+PBMC proliferation by targeting MAPK14. (A-C) pcDNA3.1-MAPK14 was successfully transfected in CD14+PBMCs. (D) pcDNA3.1-MAPK14 reversed the effects of miR-22-3p mimic on CD14 ${ }^{+} \mathrm{PBMCs}$ proliferation. ${ }^{* *} \mathrm{P}<0.01 \mathrm{vs}$. pcDNA3.1 or miR-NC mimic + pcDNA3.1. ${ }^{\&}$ P $<0.05$ vs. miR-22-3p mimic + pcDNA3.1. OP, osteoporosis; miR, microRNA; PBMCs, peripheral blood mononuclear cells; M-CSF, macrophage colony stimulating factor; RANKL, receptor activator of nuclear factor- $\mathrm{kB}$ ligand; $\mathrm{NC}$, negative control.

volunteers and patients with postmenopausal OP. The results from RT-qPCR demonstrated that miR-22-3p expression was significantly lower in patients with postmenopausal OP compared with healthy volunteers (Fig. 1A). Furthermore, miR-22-3p expression level was significantly lower in CD14 ${ }^{+}$PBMCs treated with M-CSF and RANKL for 6 days compared with those untreated (Fig. 1B). These results suggested that miR-22-3p may be involved in the development of OP.

miR-22-3p targets the expression of MAPK14. MAPK14 was predicted to be a target gene for miR-22-3p by TargetScan. The predicted pairing between MAPK14 3'UTR and miR-22-3p is presented in Fig. 2A. In addition, compared with miR-NC mimic group, miR-22-3p mimic significantly increased miR-22-3p expression level in CD14 ${ }^{+}$BBMCs (Fig. 2B). Subsequently, the interaction between miR-22-3p and MAPK14 in CD14+PBMCs was detected by dual luciferase reporter assay. Compared with miR-NC mimic group, miR-22-3p mimic significantly decreased the luciferase activity of the WT-MAPK14 but not MUT-MAPK14 (Fig. 2C). These findings confirmed the potential interaction between miR-22-3p and MAPK14.

MAPK14 expression in participants and $C D 14^{+} P B M C s$. To explore the effects of MAPK14 during the development of OP, MAPK14 expression was evaluated in healthy volunteers and patients with postmenopausal OP. The results from RT-qPCR demonstrated that MAPK14 expression was significantly higher in patients with postmenopausal OP compared with healthy volunteers (Fig. 3A). Furthermore, Pearson correlation analysis demonstrated that miR-22-3p and MAPK14 expression levels were negatively correlated in patients with postmenopausal OP (Fig. 3B).

In addition, results from RT-qPCR showed that MAPK14 expression level was significantly higher in CD14+PBMCs treated with M-CSF and RANKL for 6 days compared with those untreated (Fig. 3C). These findings suggested that MAPK14 may be implicated in the development of OP.

miR-22-3p mimic inhibits $C D 14^{+} P B M C$ proliferation by targeting $M A P K 14$. Prior to the assessment of cell proliferation, CD14 ${ }^{+}$BMCs were transfected with pcDNA3.1-MAPK14 to overexpress MAPK14. The results from RT-qPCR and western blotting demonstrated that the mRNA (Fig. 4A) and protein (Fig. 4B and C) expression of MAPK14 was significantly increased following transfection with pcDNA3.1-MAPK14 in $\mathrm{CD} 14^{+} \mathrm{PBMC}$ compared with pcDNA3.1 group, which confirmed the successful transfection of pcDNA3.1-MAPK14 into $\mathrm{CD} 14^{+} \mathrm{PBMCs}$.

The results from CCK-8 showed that at day 6 of incubation with M-CSF and RANKL, miR-22-3p mimic significantly decreased CD14+PBMC proliferation compared with miR-NC mimic + pcDNA3.1 group, which was significantly rescued following co-transfection with pcDNA3.1-MAPK14 (Fig. 4D). 
A

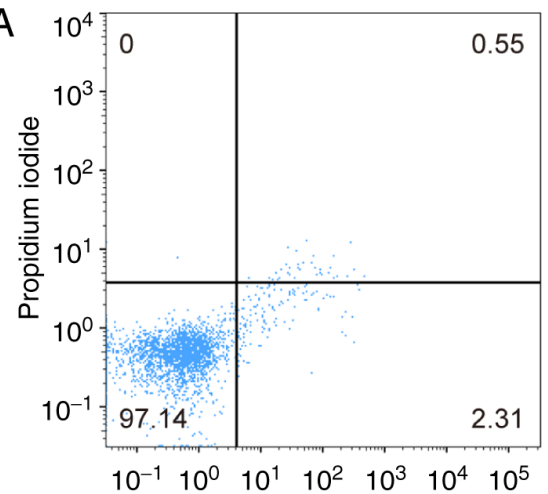

miR-NC mimic

pcDNA3.1

miR-22-3p mimic

pCDNA3.1-MAPK14
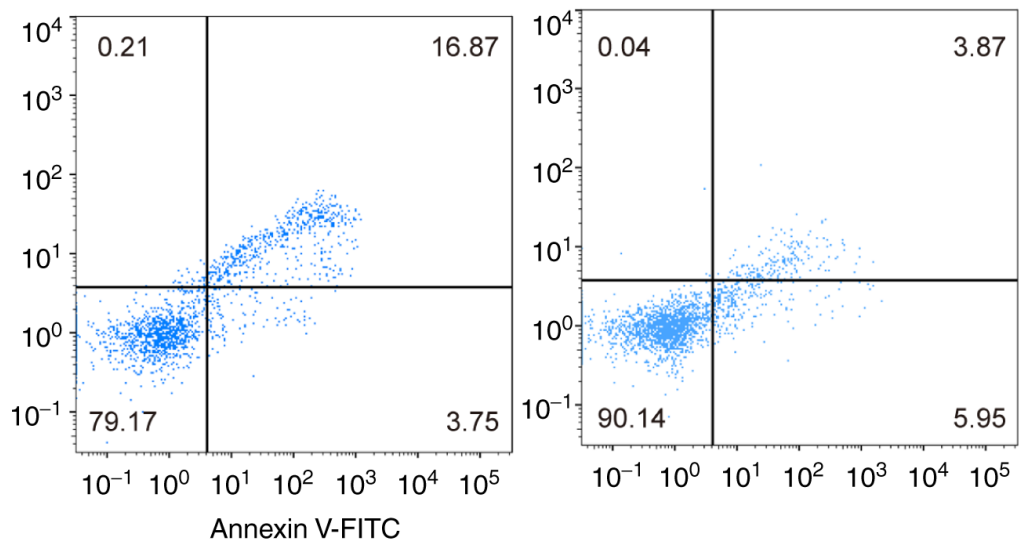

$-$

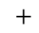

$+$

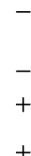

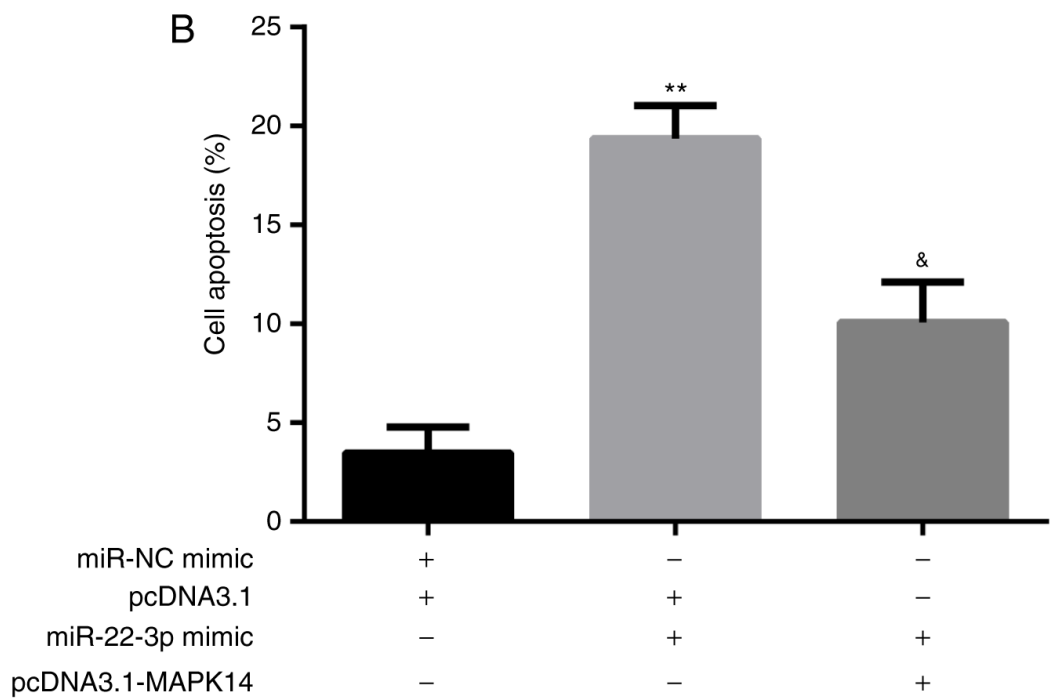

Figure 5. miR-22-3p mimic promotes CD14+PBMCs apoptosis by targeting MAPK14. (A and B) pcDNA3.1-MAPK14 reversed miR-22-3p mimic-induced CD14+PBMC apoptosis. ${ }^{* *} \mathrm{P}<0.01$ vs. pcDNA3.1 or miR-NC mimic + pcDNA3.1. ${ }^{\&} \mathrm{P}<0.05$ vs. miR-22-3p mimic + pcDNA3.1. miR, microRNA; PBMCs, peripheral blood mononuclear cells; NC, negative control.

miR-22-3p mimic promotes $C D 14^{+} P B M C$ apoptosis by targeting MAPK14. The results from flow cytometry showed that at day 6 of incubation of M-CSF and RANKL, miR-22-3p mimic significantly increased CD14+PBMC apoptosis compared with miR-NC mimic + pcDNA3.1 group, which was significantly rescued following co-transfection with pcDNA3.1-MAPK14 (Fig. 5A and B).

miR-22-3p mimic inhibits $C D 14^{+} P B M C$ differentiation by targeting MAPK14. The results from RT-qPCR and western blotting exhibited that at day 6 after incubation with M-CSF and RANKL, miR-22-3p mimic significantly decreased the mRNA and protein expression of the osteoclast markers, including TRAP, NFATC1, and CTSK, compared with miR-NC mimic + pcDNA3.1 group. These observations were reversed following co-transfection with pcDNA3.1-MAPK14 (Fig. 6A-C). These findings indicated that miR-22-3p mimic may attenuate OP via reducing the osteoclastic proliferation and differentiation, while inducing osteoclastic apoptosis by targeting MAPK14.
miR-22-3p mimic inhibits $N F-\kappa B$ activity by targeting $M A P K 14$. The results from western blotting showed that, at day 6 after incubation with M-CSF and RANKL, miR-22-3p mimic decreased the protein expression of $N F-\kappa B$ p-p65 compared with miR-NC mimic + pcDNA3.1 group. These observations were reversed following co-transfection with pcDNA3.1-MAPK14 (Fig. 7A and B). These results suggested that miR-22-3p mimic may attenuate OP by targeting MAPK14 and inactivating $\mathrm{NF}-\kappa \mathrm{B}$ p 65 .

\section{Discussion}

Abnormal expression of miRNAs has been shown to be associated with the process of OP (16), and certain miRNAs are implicated in multiple biological processes, including bone remolding (25). For example, estrogen can downregulate miR-21 biogenesis and upregulate its target gene Fas ligand, inhibiting therefore osteoclastogenesis and inducing osteoclastic apoptosis $(26,27)$. Furthermore, miR-223, of which expression is decreased during RAW264.7 osteoclast differentiation, represses 
A

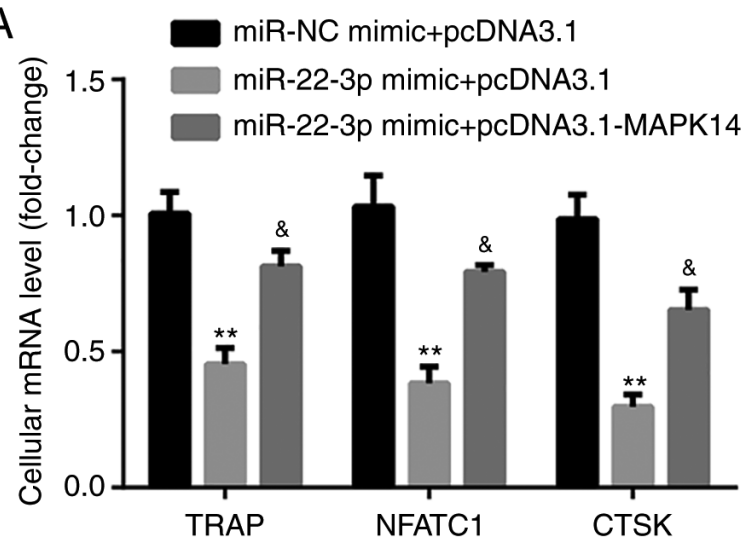

B

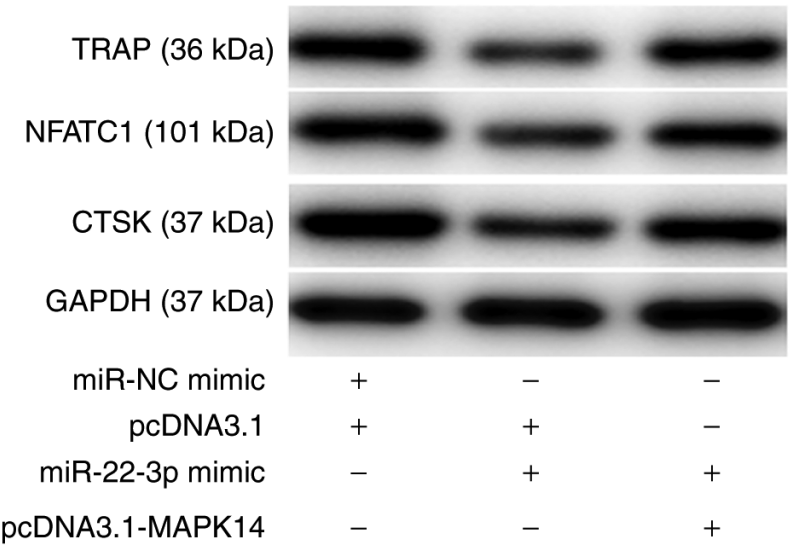

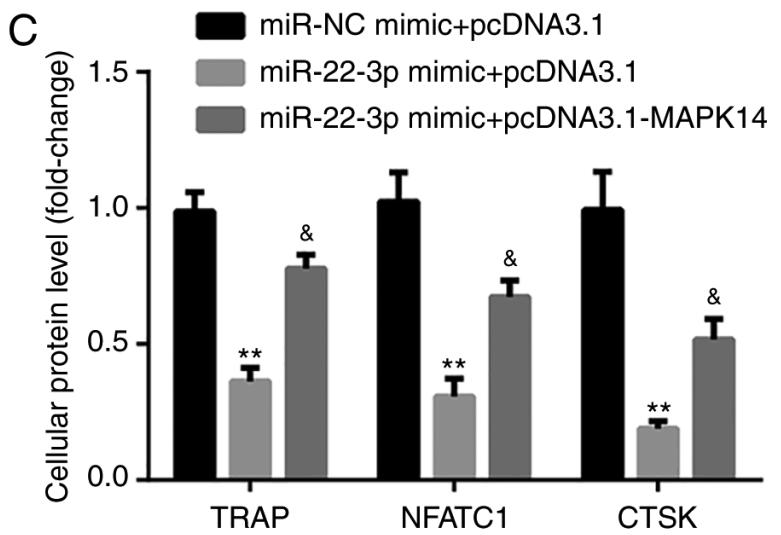

Figure 6. miR-22-3p mimic inhibits CD14 PBMC differentiation by targeting MAPK14. (A-C) pcDNA3.1-MAPK14 reversed miR-22-3p mimic-induced CD14+PBMCs decreased differentiation. ${ }^{* *} \mathrm{P}<0.01$ vs. pcDNA3.1 or miR-NC mimic + pcDNA3.1. ${ }^{\circ} \mathrm{P}<0.05$ vs. miR-22-3p mimic + pcDNA3.1. miR, microRNA; PBMCs, peripheral blood mononuclear cells; NC, negative control; TRAP, tartrate resistant acid phosphatase; NFATC1, nuclear factor of activated T-cells; CTSK, cathepsin K.

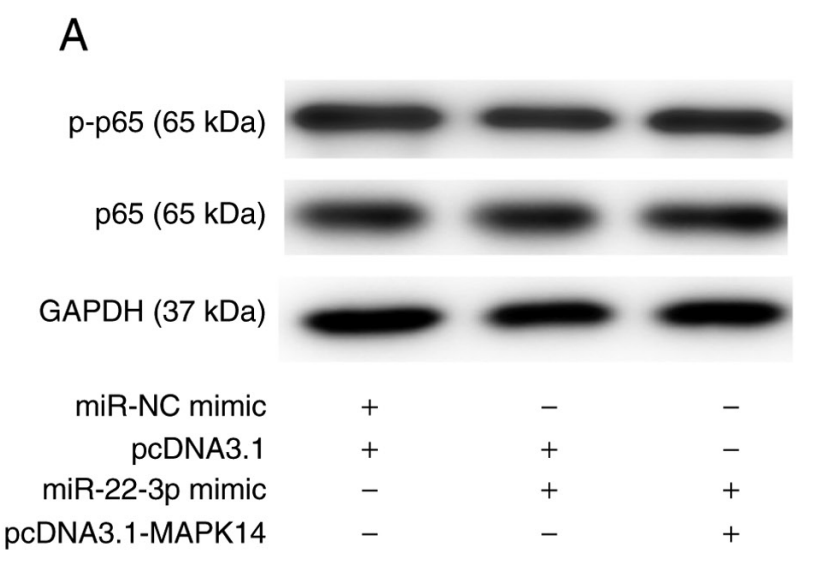

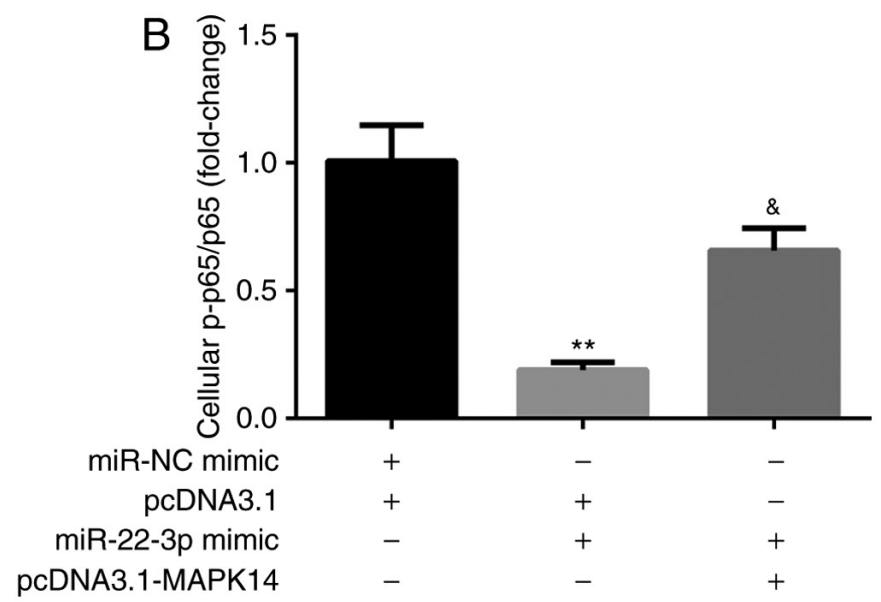

Figure 7. miR-22-3p mimic inactivates NF- $\kappa$ B by targeting MAPK14. (A and B) pcDNA3.1-MAPK14 reversed miR-22-3p mimic-induced inactivation of $\mathrm{NF}-\kappa \mathrm{B}$ p65 in $\mathrm{CD} 14^{+} \mathrm{PBMCs}{ }^{* *} \mathrm{P}<0.01$ vs. pcDNA3.1 or miR-NC mimic + pcDNA3.1. ${ }^{\circledR} \mathrm{P}<0.05$ vs. miR-22-3p mimic + pcDNA3.1. miR, microRNA; PBMCs, peripheral blood mononuclear cells; NC, negative control; p, phosphorylated.

osteoclastogenesis by targeting nuclear factor I A $(28,29)$. In addition, Linc02349 can induce the osteogenesis of human umbilical cord-derived stem cells by sponging miR-25-3p and miR-33b-5p (30). The present study aimed therefore to determine the effect of miR-22-3p on OP in osteoclast cells. It was demonstrated that miR-22-3p inactivated $\mathrm{p} 38 / \mathrm{NF}-\kappa \mathrm{B}$ pathway by MAPK14 inhibition, thus inhibiting osteoclastic proliferation and differentiation, while promoting osteoclastic apoptosis.

Originally, the present study demonstrated that miR-22-3p expression was lower in patients with postmenopausal OP and CD14 ${ }^{+} \mathrm{PBMC}$ s treated with M-CSF and RANKL for 6 days 
compared with healthy volunteers and untreated CD14+PBMCs, respectively. These findings suggested the involvement of miR-22-3p in the development of OP, which was consistent with a previous study reporting miR-22-3p downregulation during osteoclast differentiation (17). Subsequently, the target genes for miR-22-3p had to be investigated.

In the present study, MAPK14 was verified as a target of miR-22-3p in CD14+PBMCs. The p38 MAPKs represent a class of four paralogous mammalian genes, including p38 $\alpha$ /MAPK14, p38 $\beta /$ MAPK11, p38 $\gamma /$ MAPK12 and p38d/MAPK13, which together with ERK1/2 and ERK5 and the c-Jun N-terminal kinases, belong to a wider family of serine-threonine and tyrosine kinases that regulate numerous cellular processes (31). Activated p38 MAPKs can phosphorylate and activate 200-300 downstream targets (32). In addition, p38 MAPK14/11 controls the entry into primitive endoderm differentiation during the development of preimplantation mouse embryo (33). Furthermore, p38 $\alpha$ MAPK can promote bone loss. For example, specific p38 $\alpha$ inhibitors can prevent bone loss in postmenopausal OP (34), p38 $\alpha$ MAPK promotes bone loss induced by ovariectomy via increasing RANKL in osteoblast lineage cells (35), and p38 $\alpha$ MAPK induces the proliferation and differentiation of osteoclast progenitors as well as bone remodeling (36). Similarly, the present study demonstrated that MAPK14 expression was higher in patients with postmenopausal OP and CD14+PBMCs treated with M-CSF and RANKL for 6 days compared with healthy volunteers and untreated CD14+PBMCs, respectively.

Subsequently, the present study showed that miR-22-3p mimic significantly decreased osteoclastic proliferation and induced osteoclastic apoptosis by targeting MAPK14. Furthermore, the expression of the osteoclast markers TRAP, NFATC1, and CTSK (37) was significantly decreased by miR-22-3p mimic, which was rescued following MAPK14 overexpression. These results demonstrated the involvement of miR-22-3p and MAPK14 in osteoclastic proliferation, apoptosis and differentiation. Although a previous study reported that miR-22-3p targets MAPK14 in Huntington's disease (38), the present study verified for the first time their relationship in $\mathrm{OP}$, which is of great importance. However, the effects of miR22-3p and MAPK14 in the regulation of the potential signaling pathways are unknown.

As functional cytokines during osteoclastic differentiation in mammals, M-CSF and RANKL can activate NF- $\mathrm{BB}$ pathway, which in turn induces the osteoclastic survival and differentiation $(39,40)$. The present study reported that miR-22-3p mimic could inactivate $N F-\kappa B$ p65 by targeting MAPK14.

The current study presented the following limitations: i) Data on resorptive activity and images of osteoclast maturation were lacking; ii) in vivo experiments were lacking; and iii) miRNA-22-3p downregulation experiments to verify the present findings were missing. Future investigation will address these limitations.

In summary, the present study demonstrated that miR-22-3p mimic could attenuate OP via reducing osteoclastic proliferation and differentiation, while inducing osteoclastic apoptosis by targeting MAPK14 and inactivating $\mathrm{NF}-\kappa \mathrm{B}$. These findings may provide a potential therapeutic target for patients with OP.

\section{Acknowledgements}

Not applicable.

\section{Funding}

The present study was supported by the Chongqing Medical Research Program (grant no. zy201602149).

\section{Availability of materials and methods}

The datasets used and/or analyzed during the current study are available from the corresponding author on reasonable request.

\section{Authors' contributions}

$\mathrm{XJ}, \mathrm{MY}$ and WH performed the experiments and data analysis. $\mathrm{SC}$ conceived the project, supervised the experiments and data analysis, and prepared the manuscript. XJ, MY, WH and SC confirmed the authenticity of all the raw data. All authors have read and approved the final manuscript.

\section{Ethics approval and consent to participate}

This study was approved by the Ethics Committee of Chongqing Public Health Medical Center (approval no. CMC20180106). Each participant provided signed informed consent prior to the beginning of the study.

\section{Patient consent for publication}

Not applicable.

\section{Competing interests}

The authors declare that there have no competing interests.

\section{References}

1. Cosman F, de Beur SJ, LeBoff MS, Lewiecki EM, Tanner B, Randall S and Lindsay R: Erratum to: Clinician's guide to prevention and treatment of osteoporosis. Osteoporos Int 26: 2045-2047, 2015.

2. Nguyen BN, Hoshino H, Togawa D and Matsuyama Y: Cortical thickness index of the proximal femur: A radiographic parameter for preliminary assessment of bone mineral density and osteoporosis status in the age 50 years and over population. Clin Orthop Surg 10: 149-156, 2018.

3. Xiao W, Wang Y, Pacios S, Li S and Graves DT: Cellular and molecular aspects of bone remodeling. Front Oral Biol 18: 9-16, 2016.

4. Karsenty G: Transcriptional control of skeletogenesis. Annu Rev Genomics Hum Genet 9: 183-196, 2008.

5. Teitelbaum SL: Bone resorption by osteoclasts. Science 289: 1504-1508, 2000.

6. Manolagas SC: From estrogen-centric to aging and oxidative stress: A revised perspective of the pathogenesis of osteoporosis. Endocr Rev 31: 266-300, 2010.

7. Rachner TD, Khosla S and Hofbauer LC: Osteoporosis: Now and the future. Lancet 377: 1276-1287, 2011.

8. Landgraf P, Rusu M, Sheridan R, Sewer A, Iovino N, Aravin A, Pfeffer S, Rice A, Kamphorst AO, Landthaler M, et al: A mammalian microRNA expression atlas based on small RNA library sequencing. Cell 129: 1401-1414, 2007.

9. Gámez B, Rodriguez-Carballo E and Ventura F: MicroRNAs and post-transcriptional regulation of skeletal development. J Mol Endocrinol 52: R179-R197, 2014. 
10. Lian JB, Stein GS, van Wijnen AJ, Stein JL, Hassan MQ, Gaur T and Zhang Y: MicroRNA control of bone formation and homeostasis. Nat Rev Endocrinol 8: 212-227, 2012.

11. Mizuno Y, Yagi K, Tokuzawa Y, Kanesaki-Yatsuka Y, Suda T, Katagiri T, Fukuda T, Maruyama M, Okuda A, Amemiya T, et al: miR-125b inhibits osteoblastic differentiation by downregulation of cell proliferation. Biochem Biophys Res Commun 368: 267-272, 2008.

12. Wang FS, Chuang PC, Lin CL, Chen MW, Ke HJ, Chang YH, Chen YS, Wu SL and Ko JY: MicroRNA-29a protects against glucocorticoid-induced bone loss and fragility in rats by orchestrating bone acquisition and resorption. Arthritis Rheum 65: $1530-1540,2013$

13. Kahai S, Lee SC, Lee DY, Yang J, Li M, Wang CH, Jiang Z, Zhang Y, Peng C and Yang BB: MicroRNA miR-378 regulates nephronectin expression modulating osteoblast differentiation by targeting GalNT-7. PLoS One 4: e7535, 2009.

14. Xia Z, Chen C, Chen P, Xie H and Luo X: MicroRNAs and their roles in osteoclast differentiation. Front Med 5: 414-419, 2011.

15. van Wijnen AJ, van de Peppel J, van Leeuwen JP, Lian JB, Stein GS, Westendorf JJ, Oursler MJ, Im HJ, Taipaleenmäki H, Hesse E, et al: MicroRNA functions in osteogenesis and dysfunctions in osteoporosis. Curr Osteoporos Rep 11: 72-82, 2013.

16. Weilner S, Skalicky S, Salzer B, Keider V, Wagner M, Hildner F Gabriel C, Dovjak P, Pietschmann P, Grillari-Voglauer R, et al: Differentially circulating miRNAs after recent osteoporotic fractures can influence osteogenic differentiation. Bone 79 : 43-51, 2015.

17. Ma Y, Shan Z, Ma J, Wang Q, Chu J, Xu P, Qin A and Fan S: Validation of downregulated microRNAs during osteoclast formation and osteoporosis progression. Mol Med Rep 13: 2273-2280, 2016.

18. Mäkitie RE, Hackl M, Niinimäki R, Kakko S, Grillari J and Mäkitie O: Altered microRNA profile in osteoporosis caused by impaired WNT signaling. J Clin Endocrinol Metab 103: 1985-1996, 2018

19. Zhang X, Wang Y, Zhao H, Han X, Zhao T, Qu P, Li G and Wang W: Extracellular vesicle-encapsulated miR-22-3p from bone marrow mesenchymal stem cell promotes osteogenic differentiation via FTO inhibition. Stem Cell Res Ther 11: 227, 2020.

20. World Health Organization: Assessment of fracture risk and its application to screening for postmenopausal osteoporosis. Report of a WHO Study Group. World Health Organ Tech Rep Ser 843: 1-129, 1994.

21. Heming way F, Cheng X, Knowles HJ, Estrada FM, Gordon S and Athanasou NA: In vitro generation of mature human osteoclasts. Calcif Tissue Int 89: 389-395, 2011.

22. Sørensen MG, Henriksen K, Schaller S, Henriksen DB, Nielsen FC, Dziegiel MH and Karsdal MA: Characterization of osteoclasts derived from $\mathrm{CD} 14^{+}$monocytes isolated from peripheral blood. J Bone Miner Metab 25: 36-45, 2007.

23. Livak KJ and Schmittgen TD: Analysis of relative gene expression data using real-time quantitative PCR and the 2(-Delta Delta C(T)) Method. Methods 25: 402-408, 2001.

24. Cheng P, Chen C, He HB, Hu R, Zhou HD, Xie H, Zhu W, Dai RC, Wu XP, Liao EY, et al: miR-148a regulates osteoclastogenesis by targeting V-maf musculoaponeurotic fibrosarcoma oncogene homolog B. J Bone Miner Res 28: 1180-1190, 2013.
25. Jing D, Hao J, Shen Y, Tang G, Li ML, Huang SH and Zhao ZH: The role of microRNAs in bone remodeling. Int J Oral Sci 7: 131-143, 2015.

26. García Palacios V, Robinson LJ, Borysenko CW, Lehmann T, Kalla SE and Blair HC: Negative regulation of RANKL-induced osteoclastic differentiation in RAW264.7 cells by estrogen and phytoestrogens. J Biol Chem 280: 13720-13727, 2005.

27. Sugatani T and Hruska KA: Down-regulation of miR-21 biogenesis by estrogen action contributes to osteoclastic apoptosis. J Cell Biochem 114: 1217-1222, 2013.

28. Kagiya T and Nakamura S: Expression profiling of microRNAs in RAW264.7 cells treated with a combination of tumor necrosis factor alpha and RANKL during osteoclast differentiation. J Periodontal Res 48: 373-385, 2013.

29. Shibuya H, Nakasa T, Adachi N, Nagata Y, Ishikawa M, Deie M, Suzuki O and Ochi M: Overexpression of microRNA-223 in rheumatoid arthritis synovium controls osteoclast differentiation. Mod Rheumatol 23: 674-685, 2013.

30. Cao L, Liu W, Zhong Y, Zhang Y, Gao D, He T, Liu Y, Zou Z, Mo Y, Peng S, et al: Linc02349 promotes osteogenesis of human umbilical cord-derived stem cells by acting as a competing endogenous RNA for miR-25-3p and miR-33b-5p. Cell Prolif 53: e12814, 2020

31. Cargnello M and Roux PP: Activation and function of the MAPKs and their substrates, the MAPK-activated protein kinases. Microbiol Mol Biol Rev 75: 50-83, 2011.

32. Cuadrado A and Nebreda AR: Mechanisms and functions of $\mathrm{p} 38$ MAPK signalling. Biochem J 429: 403-417, 2010.

33. Thamodaran V and Bruce AW: p38 (Mapk14/11) occupies a regulatory node governing entry into primitive endoderm differentiation during preimplantation mouse embryo development. Open Biol 6: 160190, 2016.

34. Caverzasio J, Higgins L and Ammann P: Prevention of trabecular bone loss induced by estrogen deficiency by a selective p38alpha inhibitor. J Bone Miner Res 23: 1389-1397, 2008

35. Thouverey $\mathrm{C}$ and Caverzasio J: Ablation of $\mathrm{p} 38 \alpha$ MAPK signaling in osteoblast lineage cells protects mice from bone loss induced by estrogen deficiency. Endocrinology 156: 4377-4387, 2015

36. Cong Q, Jia H, Li P, Qiu S, Yeh J, Wang Y, Zhang ZL, Ao J, $\mathrm{Li} \mathrm{B}$ and Liu H: p38 $\alpha$ MAPK regulates proliferation and differentiation of osteoclast progenitors and bone remodeling in an aging-dependent manner. Sci Rep 7: 45964, 2017.

37. Boyle WJ, Simonet WS and Lacey DL: Osteoclast differentiation and activation. Nature 423: 337-342, 2003.

38. Jovicic A, Zaldivar Jolissaint JF, Moser R, Silva Santos MF and Luthi-Carter R: MicroRNA-22 (miR-22) overexpression is neuroprotective via general anti-apoptotic effects and may also target specific Huntington's disease-related mechanisms. PLoS One 8: e54222, 2013.

39. Kwon M, Kim JM, Lee K, Park SY, Lim HS, Kim T and Jeong D: Synchronized cell cycle arrest promotes osteoclast differentiation. Int J Mol Sci 17: 1292, 2016.

40. Boyce BF: Advances in osteoclast biology reveal potential new drug targets and new roles for osteoclasts. J Bone Miner Res 28: 711-722, 2013

c) (i) $\odot$ This work is licensed under a Creative Commons Attribution-NonCommercial-NoDerivatives 4.0 International (CC BY-NC-ND 4.0) License. 